\title{
Braess's Paradox and Power-Law Nonlinearities in Five-Arc and Six-Arc Two-Terminal Networks
}

\author{
Claude M. Penchina* \\ Department of Physics, Hasbrouck Laboratory, University of Massachusetts at Amherst, Amherst Massachusetts 01003 \\ USA \\ Additional Affiliations: Department of Physics, King's College, Strand, London WC2R-2LS, UK, and Gilora Associates, \\ Flemington, New Jersey, NJ 08822, USA
}

\begin{abstract}
We extend a general network theorem of Calvert and Keady (CK) relating to the minimum number of arcs needed to guarantee the occurrence of the Braess Paradox. We rephrase the CK theorems and express our proof in the terminology of traffic networks.

CK described their theorem in relation to a two-terminal network of liquid in pipes. "Approximately stated, it is: if every relationship between flow and head difference is not a power law, with the same (power) s on each arc, given at least 6 pipes, one can arrange (lengths of) them so that Braess's paradox occurs, i.e. one can increase the conductivity of an individual pipe yet require more power to maintain the same consumptions." In relation to the original Braess situation of traffic network flows, the relationship is between flow and link-cost on a congested link.

Our extended theorem shows that 5 pipes (roads, links, arcs) arranged in a Wheatstone Bridge (WB) network (as in the original Braess network) are necessary and sufficient to produce a Braess paradox (BP) in a two-terminal network (not limited to liquid in pipes) if at least one of the five has a different conductivity law (not power s).
\end{abstract}

Keywords: Braess's paradox, network theorems, power law non-linearities, wheatstone bridge.

\section{INTRODUCTION}

Braess [1] described a "paradoxical" traffic network for which an extra road (arc, link) added with the express aim to relieve congestion, instead increased the travel time for all users. Calvert and Keady [2], hereinafter referred to as CK, restated the paradox for "physical" networks. "BRAESS'S PARADOX. The power consumed in a nonlinear network can increase if an arc's conductivity is increased with consumptions held constant." Penchina [3] restated it for electrical networks: "BRAESS'S PARADOX. Decreasing the resistance in a branch of the circuit can decrease the current at fixed input voltage, or increase the voltage at fixed input currents." Here, we restate it for traffic networks: "BRAESS'S PARADOX. Increasing the capacity ${ }^{l}$ in an arc of a network can increase the network cost at fixed demand."

CK were particularly concerned with physical twoterminal water-supply pipe networks. Here, we are concerned mainly with traffic networks. The results apply equally to both, and to any other network with "a potential function defined over the nodes and a flow defined over the arcs" [2] which satisfies Kirchhoff's rules. Examples of such networks and their corresponding terminologies are in

*Address correspondence to this author at the Department of Physics, Hasbrouck Laboratory, University of Massachusetts at Amherst, Amherst Massachusetts, 01003, USA; E-mail: penchina@physics.umass.edu

${ }^{1}$ To avoid awkward writing style, we use the words Increasing the capacity here loosely as a shorthand expression to indicate decreasing the congestion cost per unit flow.
Penchina [3], Table 1 of which is repeated here for the convenience of the reader, with an additional column for a network of mechanical springs, based on Penchina [4]. Note that in traffic networks, a node's potential (see Dial [5] p. 191) equals the "length" (total generalized cost, GC, including tolls) of the min-GC path from the origin node to it.

CK proved several theorems concerning the occurrence or non-occurrence of the Braess Paradox (hereinafter referred to as BP). Their final result: "Approximately stated, it is: if every relationship between flow and head difference is not a power law, with the same (power) s on each arc, given at least 6 pipes, one can arrange (lengths of) them so that Braess's paradsox occurs, i.e. one can increase the conductivity of an individual pipe yet require more power to maintain the same consumptions ${ }^{2}$."

We extend this result by showing that the required number $v$ of pipes is $v>=5$. The equality applies only for the Wheatstone Bridge (hereinafter referred to as $\mathbf{W B}$ ) arrangement of Fig. (1A), as in the original network of Braess [1]. For the convenience of the reader interested in traffic/transportation we rephrase the CK theorems and describe our proof in traffic network terminology instead of the terminology of water-pipe networks.

To summarize, our extended theorem shows that 5 pipes (roads, links, arcs) arranged in a $W B$ (as in the original

\footnotetext{
${ }^{2}$ The need for not all pipes with power law s (necessary condition) can be seen intuitively from the fact that if all had power law s, the ratio of marginal costs to average costs would be the same for all pipes. The sufficient condition is more complicated to explain.
} 
Table 1. Equivalent Terms for Various Networks

\begin{tabular}{|c|c|c|c|c|c|}
\hline Network & Traffic & Water & Electrical & Thermal & Spring \\
\hline Node, Vertex & Intersection & Joint & $\begin{array}{l}\text { Junction, } \\
\text { Node }\end{array}$ & Junction & Connection \\
\hline Arc, Edge, Link & $\begin{array}{l}\text { Road, Link, } \\
\text { Arc }\end{array}$ & Pipe & Branch & & $\begin{array}{l}\text { Spring } \\
\text { String }\end{array}$ \\
\hline Potential & Time or GC & Head $=p$ & $\begin{array}{c}\text { Voltage } \\
=\mathrm{V}\end{array}$ & $\begin{array}{c}\text { Temperature } \\
=\mathrm{T}\end{array}$ & Height \\
\hline $\begin{array}{c}\text { Reference } \\
\text { Potential (zero) }\end{array}$ & Origin & Sea Level & $\begin{array}{l}\text { Ground, } \\
\text { Earth }\end{array}$ & $\begin{array}{l}\text { Heat Sink, } \\
\text { Reservoir }\end{array}$ & $\begin{array}{l}\text { Height of } \\
\text { Support }\end{array}$ \\
\hline Flow & Traffic Flow & $\begin{array}{c}\text { Water Flow } \\
\quad=\mathrm{U}\end{array}$ & $\begin{array}{l}\text { Current } \\
\quad=\mathrm{I}\end{array}$ & $\begin{array}{l}\text { Heat-Flow } \\
\quad=\mathrm{Q}\end{array}$ & Force \\
\hline Consumptions & Demands & $\begin{array}{l}\text { Source, } \\
\text { Supply }\end{array}$ & $\begin{array}{c}\text { Input } \\
\text { Currents }\end{array}$ & Heat Input & $\begin{array}{l}\text { Weight } \\
\text { of Load }\end{array}$ \\
\hline $\begin{array}{c}\text { Power }= \\
\text { Flow } \times \Delta \text { Potential }\end{array}$ & $\begin{array}{l}\text { Vehicle Hours, } \\
\text { System Cost }\end{array}$ & Flow x $\Delta \mathrm{p}$ & $\begin{array}{l}\text { Power } \\
=\mathrm{I} \times \Delta \mathrm{V}\end{array}$ & $\mathrm{Q} \times \Delta \mathrm{T}$ & $\begin{array}{c}\text { Work }= \\
\text { Weight } \mathrm{x} \Delta \text { Height }\end{array}$ \\
\hline
\end{tabular}

In traffic networks GC represents Generalized Cost = Time plus the time-equivalent of other costs. This table repeats Table 1 of Penchina. Note: A node's potential equals the "length" (total generalized cost, GC, including tolls) of the min-GC path from the origin node to it (see Dial [5] p. 191)

Braess network) are necessary and sufficient to produce a Braess paradox in a two-terminal network if at least one of the five has a different conductivity law (not power s).

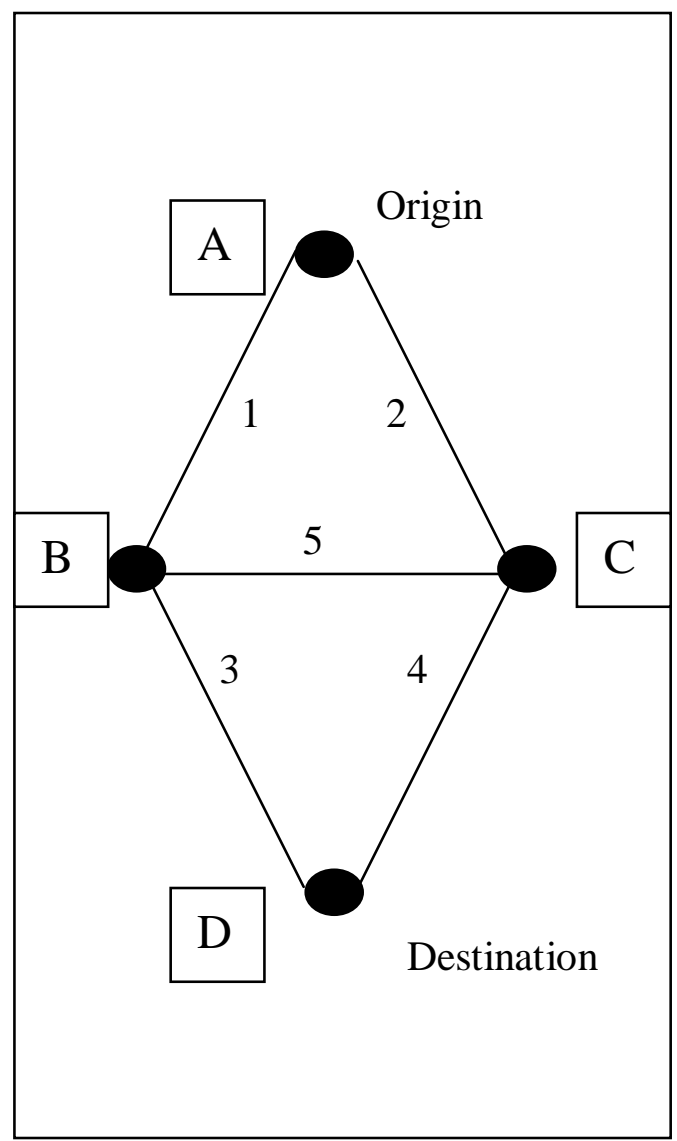

Fig. (1A). A simple, 5-arc, 1-origin, 1-destination, 2-terminal "Wheatstone Bridge" network. In the Braess [1] paradoxical network, all links are 1 way, so there are 3 paths: ABD, ACD, ABCD. The Minimal Critical Network of Penchina [7] had a two-way link (bridge) $\mathbf{B C}$, and thus a 4th path ACBD.

\section{PROOF}

Our proof of the extension of the CK result relies on four of their theorems $(1,2,11$, and 12). For full details of their theorems, the reader is referred to the original reference [2]. Here, we restate them approximately, paraphrasing them in the terminology of traffic networks.

CK Theorem 1. Deals with networks made up of arcs, a, each of which has a flow $\left(U_{a}\right)$ which is proportional to a conductivity function $\left(\sigma_{\mathrm{a}}\right)$ which is a power law function of the potential difference (link/arc cost $C_{a}$ ), with power (s) being the same positive number for each arc. i.e. the flow on arc $\mathrm{a}$ is $\mathrm{U}_{\mathrm{a}}=\mathrm{k}_{\mathrm{a}} \sigma_{\mathrm{a}}$ where $\sigma_{\mathrm{a}}\left(\mathrm{C}_{\mathrm{a}}\right)=\sigma_{\mathrm{a}}(1)\left(\mathrm{C}_{\mathrm{a}}\right)^{\mathrm{s}} \mathbf{3}$ where $\mathrm{s}$ is independent of $a$. The theorem shows that the system cost (measured, e.g., in vehicle-hours) is a non-decreasing function of $\mathrm{k}_{\mathrm{a}}$ i.e. the BP can not occur for such a power-law network.

CK Theorem 2. A converse to Theorem 1. Approximately stated, in the language of the CK abstract, (but in traffic terms): If every relationship between flow $\mathrm{U}$ and cost $\mathrm{C}$ is not a power law, with the same power s on each arc, given at least 6 arcs, one can arrange lengths of them so that Braess's paradox occurs.

CK Theorem 11. Shows that the BP can not occur in purely series-parallel networks

CK Theorem 12. Shows that "A two terminal network is series-parallel if and only if there is no embedded network having the WB configuration" (see Calvert [2] p14 and also Milchtaich [6]).

CK Theorems 11 and 12. Show that, in order for the Braess Paradox to occur in a six-arc two-terminal network, five of the arcs must form a $W B$ as illustrated in Fig. (1A).

\footnotetext{
${ }^{3}$ More precisely, CK use the equivalent of $\sigma_{a}\left(C_{a}\right)=\sigma_{a}(1) C_{a}\left|C_{a}\right|^{(s-1)}$ to deal
} with negative as well as positive potential differences $\mathrm{C}_{\mathrm{a}}$. 
Our proof proceeds by examining the effects of each possible way one can add a sixth arc to a five-arc $W B$.

There are nine ways in which the sixth arc can be added to this network, several of them equivalent, resulting in six non-equivalent independent additions. Sections 2.1 and 2.2 discuss one way each; two ways are discussed in each of sections $2.3 \& 2.4$.

\subsection{Sixth Arc in Series at D or A}

Fig. (1B) adds the sixth arc in series with the five-arc network at node D. An equivalent addition would be at node A.

If the total network flow $\mathrm{U}$ from origin A to destination $\mathrm{D}$ in the five-arc network of Fig. (1A) is $U=U_{A D}$, then we could adjust $\mathrm{C}_{\mathrm{DE}}$, the potential difference (arc cost) from $\mathrm{D}$ to $\mathrm{E}$, to produce the same flow $\mathrm{U}=\mathrm{U}_{\mathrm{AE}}$ from $\mathrm{A}$ to $\mathrm{E}$ in Fig. (1B). i.e.

$$
\mathrm{U}_{\mathrm{AE}}(\text { Fig. 1B })=\mathrm{U}_{\mathrm{AD}}(\text { Fig. 1A })=\mathrm{U}
$$

Since the sixth arc is added in series,

$$
\begin{aligned}
\mathrm{U}_{\mathrm{AD}} \text { (Fig. 1B) } & =\mathrm{U}_{\mathrm{AE}} \text { (Fig. 1B) } \\
& =\mathrm{U}_{\mathrm{AD}}(\text { Fig. 1A })
\end{aligned}
$$

so the potential differences (costs) and flows in the original five arcs are unchanged by this addition of the sixth arc in series. Hence, with a constant flow (inelastic demand flow) the added sixth arc can not produce a Braess paradox if it did not already exist for the original five-arc network. Also, the sixth arc can not eliminate the paradox if it already exists in the five-arc network.

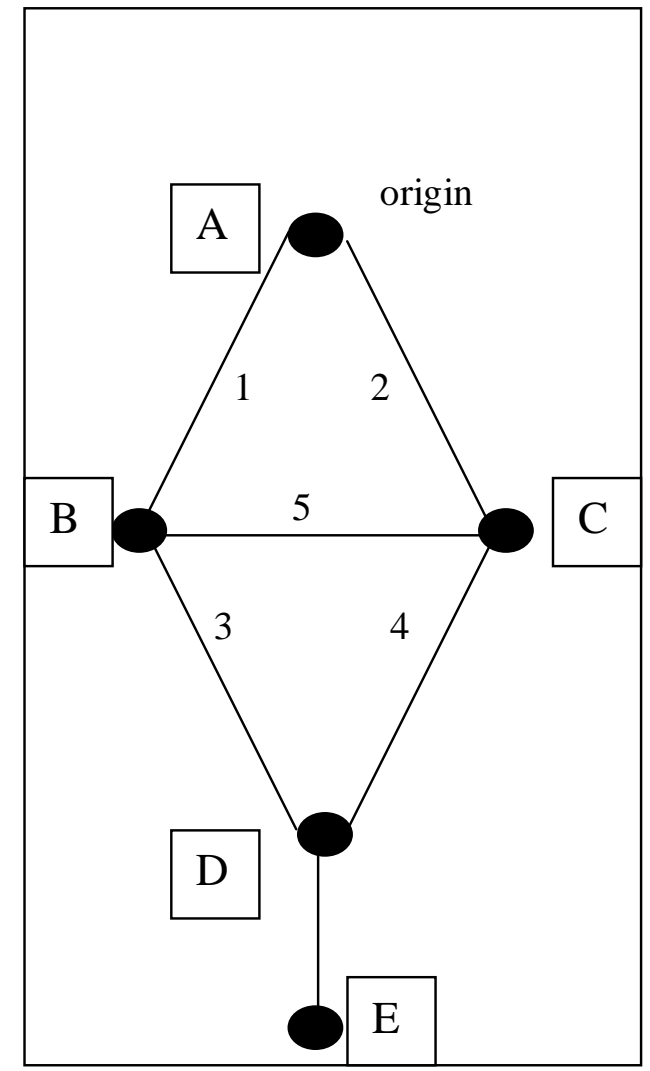

Fig. (1B). A sixth arc DE is added in series at D. Equivalently the sixth arc could be added at A. Analysis shows that this sixth arc does not affect the existence or non-existence of the paradox.
Penchina [7] showed by a graphic analysis (graph reproduced here also as Fig. (2) which uses terminology from Frank [8]), that if the Braess Paradox can not occur with inelastic demand, this implies there is no loop in the figure. Thus, the paradox can not occur for elastic demand either, even totally elastic demand i.e. even for a fixed potential from origin to destination (total user cost). So, if the BP is to occur in the six-arc network $1 \mathrm{~B}$, it must occur also in the five-arc network $1 \mathrm{~A}$.

\subsection{Sixth Arc in PARALLEL from A to D}

See Fig. (1C). For any given fixed potential difference (arc cost) $\mathrm{C}_{\mathrm{AD}}$ (equivalent to totally elastic demand) across the original network (Fig. 1A), the sixth arc AD will not affect the flows through the original five arcs. Thus, it can not affect the occurrence or non-occurrence of the BP in the original five arcs. Penchina [7] showed by a graphic analysis (graph reproduced here also as Fig. (2)), that if the BP can not occur with elastic demand, then there is no loop in the graph, and the paradox can not occur for inelastic demand either. Thus, if there is no paradox in the five-arc network, there is also no paradox in the six-arc network, whereas if there is a paradox in the five-arc network, there is also a paradox in the six-arc network

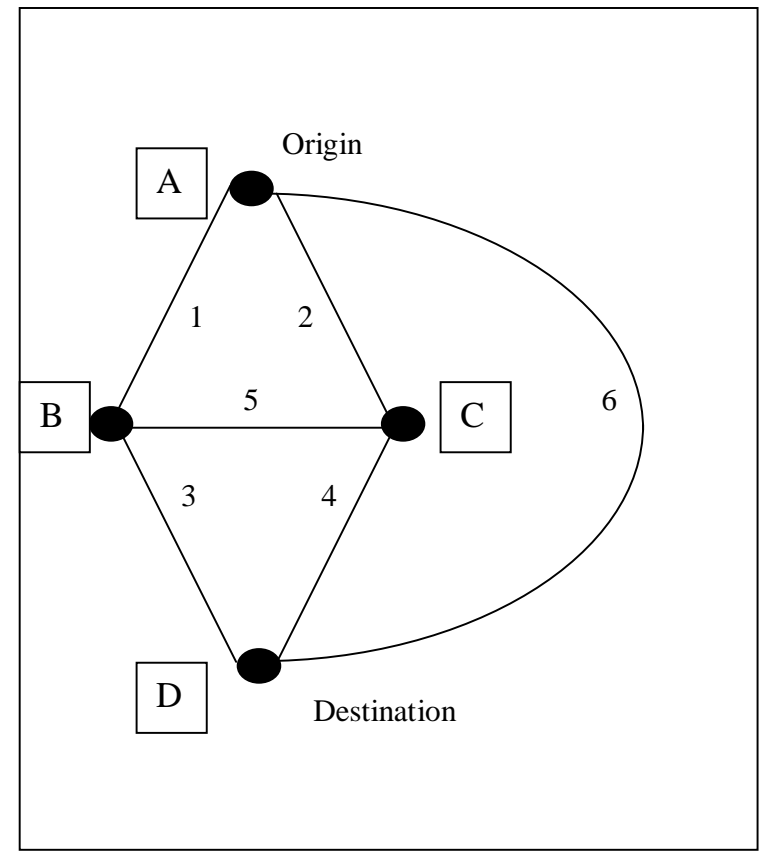

Fig. (1C). A sixth arc is added in parallel across AD. Analysis shows that this sixth arc does not affect the existence or nonexistence of the paradox.

\subsection{Sixth Arc in PARALLEL with One of the Original Five Arcs}

Fig. (1D) adds the sixth arc in parallel across arc 4. By symmetry, it is equivalent to add the sixth arc in parallel across arcs 1, 2, or 3. Another independent place to add a sixth arc is in parallel across arc 5. This is not equivalent by any simple symmetry to the addition across arc 4 . Note, however, that the reasoning to be used in our proof is not symmetry dependent; the argument applies equally well to arc 5 (as well as to $\operatorname{arcs} 1,2$, and 3 without regard to the symmetry); i.e. applies to a sixth arc connected in parallel 
with any one of the five original arcs. Thus, we treat them all together, using Fig. (1D) as our prototype.

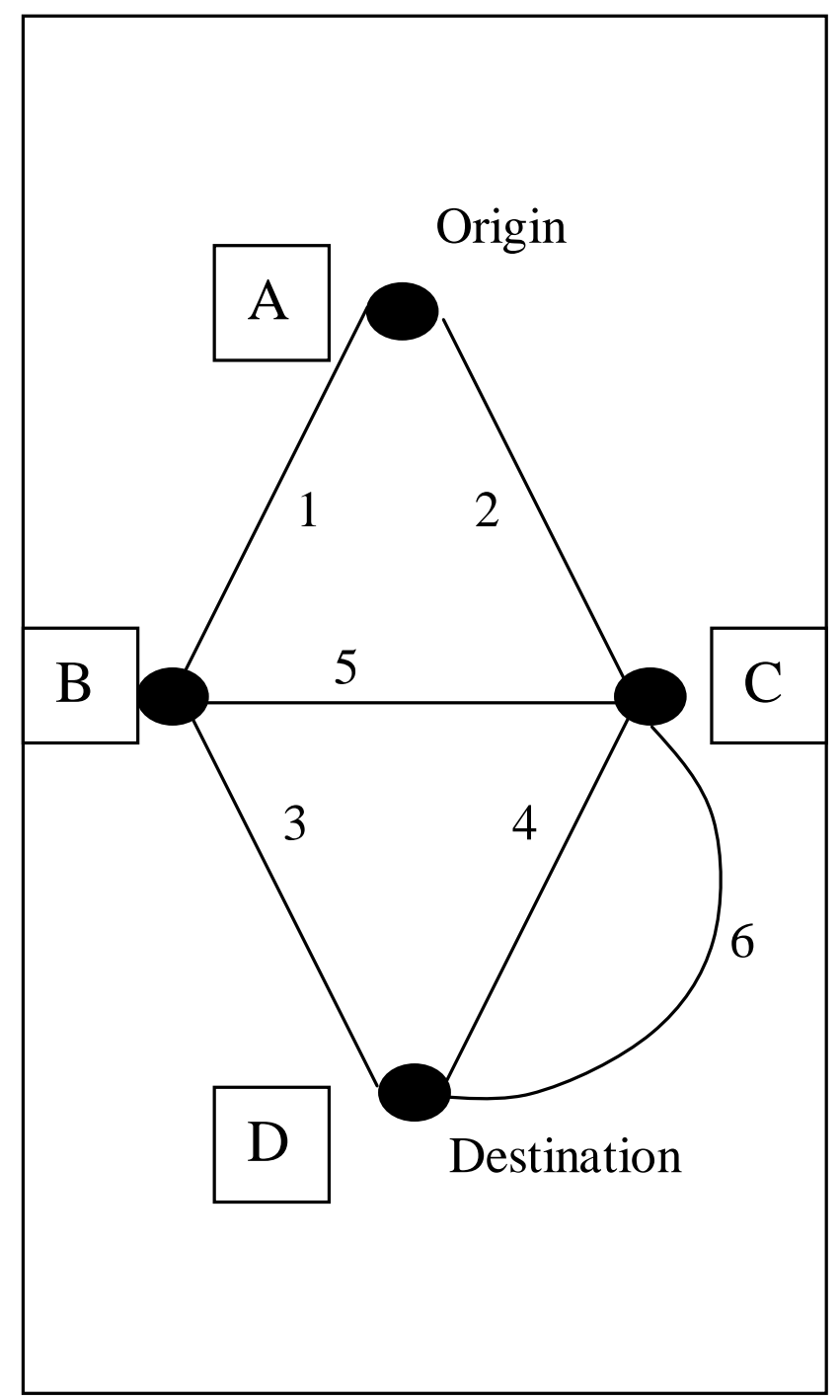

Fig. (1D). A sixth arc is added in parallel across CD Our analysis applies equally well to nodes $\mathbf{A B}, \mathbf{A C}, \mathbf{B C}$, and $\mathbf{B D}$. This analysis shows that a sixth arc with conductivity of power law s will neither cause nor prevent the Paradox. Analysis also shows that a sixth arc without power law s could cause the paradox, but it could then be produced equally well without the sixth arc if the original $\operatorname{arc}(\operatorname{arc} 4$ in this figure) between the same two nodes (nodes CD in this figure) does not have power law s.

If the conductivity functions of the original arc 4 and the added sixth arc both obey the CK condition

$$
\begin{aligned}
& \sigma_{4}\left(\mathrm{C}_{\mathrm{CD}}\right)=\sigma_{4}(1)\left(\mathrm{C}_{\mathrm{CD}}\right)^{\mathrm{s}} \\
& \sigma_{6}\left(\mathrm{C}_{6}\right)=\sigma_{6}(1)\left(\mathrm{C}_{6}\right)^{\mathrm{s}}
\end{aligned}
$$

When arcs 4 and 6 are in parallel, $\mathrm{C}_{4}=\mathrm{C}_{6}=\mathrm{C}_{\mathrm{CD}}$ so the effective conductivity function $\sigma_{\text {effective }}$ of both arcs in parallel between nodes $\mathrm{C}$ and $\mathrm{D}$ is then

$$
\sigma_{\text {effective }}\left(\mathrm{C}_{\mathrm{CD}}\right)=\left\{\sigma_{6}(1)+\sigma_{4}(1)\right\}\left(\mathrm{C}_{\mathrm{CD}}\right)^{\mathrm{s}}=\sigma_{\text {equivalent }}(1)\left(\mathrm{C}_{\mathrm{CD}}\right)^{\mathrm{s}}
$$

where we have defined an equivalent conductivity function between any two nodes as

$$
\sigma_{\text {equivalent }}(C)=\left\{\sigma_{6}(C)+\sigma_{4}(C)\right\}
$$

Thus, the effect of the two parallel arcs between $\mathrm{C}$ and $\mathrm{D}$ is the same as a single arc between $C$ and $D$ with a conductivity function that has the same power law dependence on cost as before.

If either the original arc 4 or the added sixth arc (or both) has a different conductivity function (not simply a power s), then the parallel combination also does not have a simple power law s. This parallel connection produces the type of six-arc network which $\mathrm{CK}$ have shown can be arranged to have a BP. Note, however, that it is equivalent to a five-arc $W B$ network in which one arc, arc 4, does not have the simple power-law s.

Thus, if one can produce a BP with these six arcs, one can also produce it with five arcs, at least one of which does not have simply power law s.

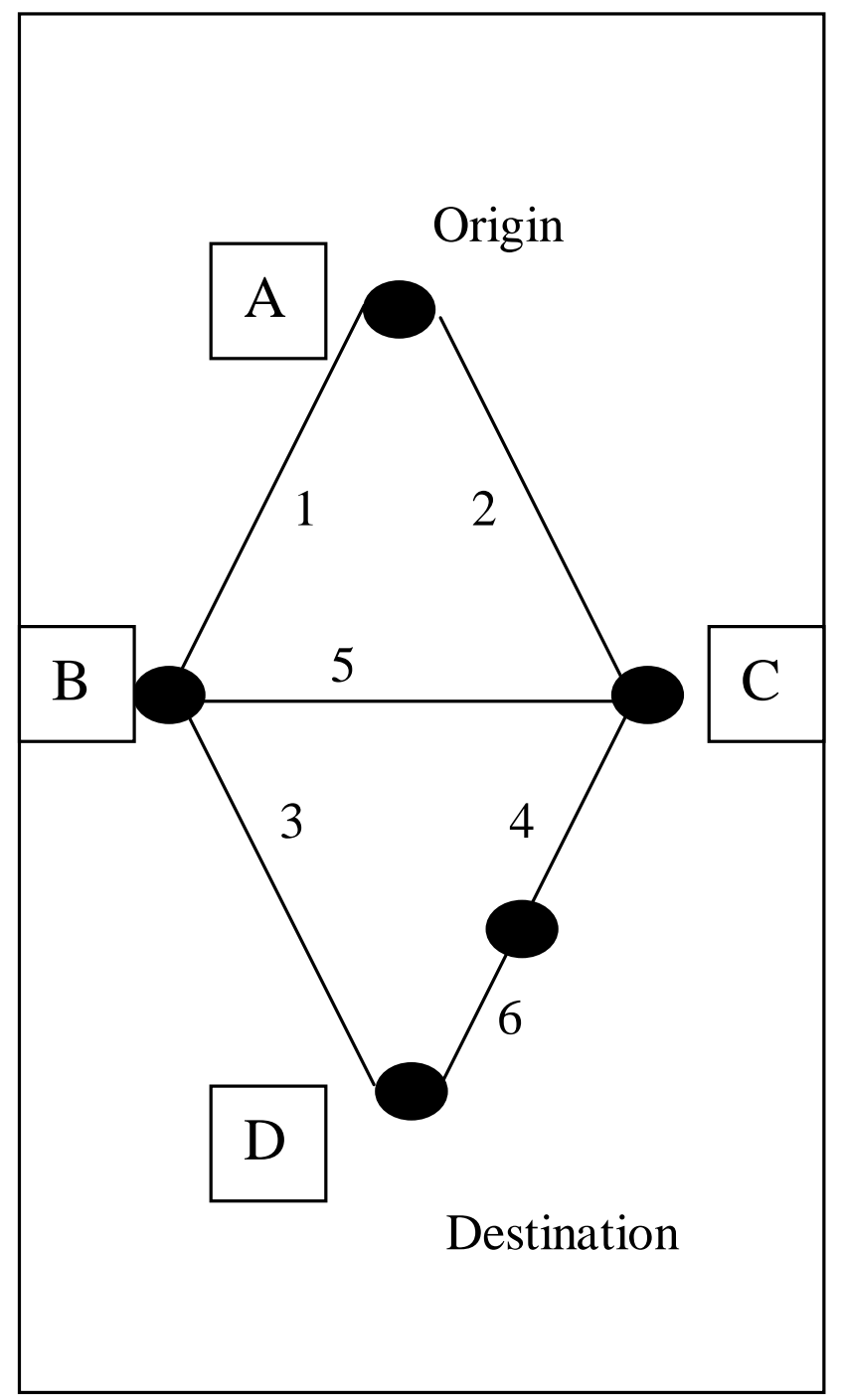

Fig. (1E). A sixth arc is added in series with arc 4 between nodes CD. Our analysis applies equally well to nodes $\mathbf{A B}, \mathbf{A C}, \mathbf{B C}$, and BD. This analysis shows that a sixth arc with conductivity of power law s will neither cause nor prevent the Paradox. Analysis also shows that a sixth arc without power law s could cause the paradox, but it could then be produced equally well without the sixth arc if the original arc (arc 4 in this figure) between the same two nodes (nodes CD in this figure) does not have power law s. 


\section{The Braess Paradox in Minimal Critical Network: User Cost vs Network Flow}

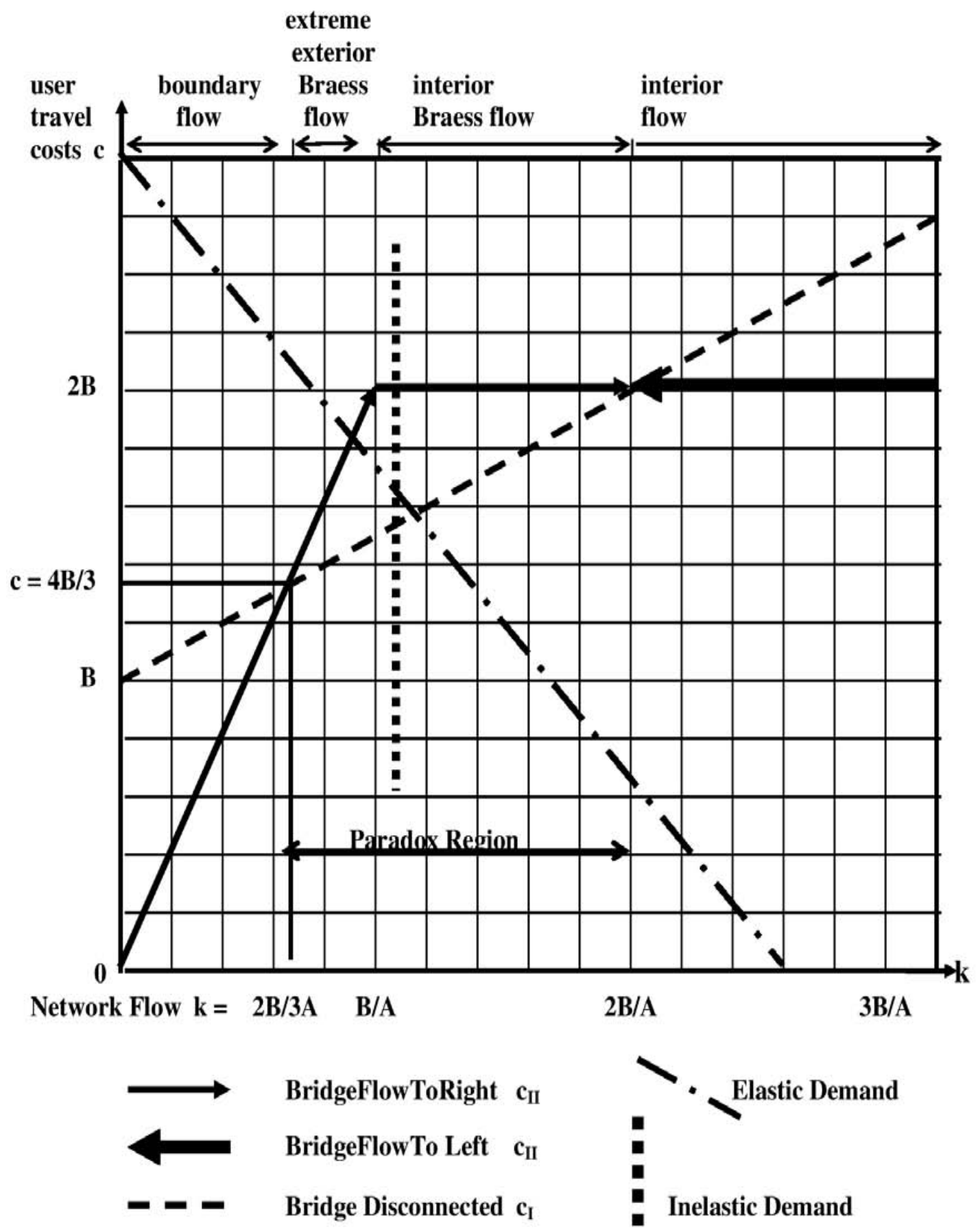

Fig. (2). The graphical analysis of the Braess paradox in a Minimal Critical Traffic Network, reproduced from Fig. (2) of reference [7] Claude M. Penchina, "Braess Paradox: Maximal Penalty in a Minimal Critical Network", Transportation Research, vol. A31(5), pp. 379-388, 1997. Two lines have been added to illustrate an inelastic demand and a simple linear elastic demand, both passing through the "loop" region formed between the curves of cost $v s$ flow with and without the "bridge" ( $\operatorname{arc} 5$ in Fig. (1)) of the Wheatstone Bridge. For the piecewise linear cost functions of Fig. (2), the loop region is a triangle. For more general cost functions, the loop region would be a more general figure. For more general elastic demands, the dash-dot line would not be straight. Adding the bridge is equivalent to using a large (infinite if the bridge has 0 cost) value of $\mathrm{k}_{\mathrm{BC}}$. Removing the bridge is equivalent to using a small (zero) value of $\mathrm{kBC}$. This analysis shows the importance of the "loop" between the graphs of the cost functions, with and without the bridge. The cost with/without the bridge is found at the intersection of the demand function with the solid/dashed line cost functions. The paradox occurs whenever the graph of the demand function (whether elastic or inelastic) crosses through this loop so that the cost is higher with the bridge than without. The labels at the top, for the various flow regions, are in the terminology of Frank [8]. See Table 1 for the equivalent variables in networks other than traffic networks. 
Because the CK theorem applies for any arbitrary arcs 4 and 6 (with the appropriate restrictions on power law dependence), in the final equivalent five arc network, arc 4 is also an arbitrary arc with the power law of the combination (power $\mathrm{s}$ if arc 4 and arc 6 each have power s, and not simply power $s$ if either arc 4 , or arc 6 , or both are not simply power s)

\subsection{Sixth Arc in SERIES with One of the Original Five Ares}

Fig. (1E) adds the sixth arc in series with arc 4 between nodes C and D. By symmetry, it is equivalent to add the sixth arc in series with arcs 1,2 , or 3 . Another independent place to add a sixth arc is in series with arc 5. This is not equivalent by any simple symmetry to the addition in series with arc 4 . Note, however, that the reasoning to be used in our proof is not symmetry dependent; the argument applies equally well to arc 5 (as well as to arcs 1,2, and 3 without regard to the symmetry); i.e. applies to a sixth arc connected in series with any one of the five original arcs. Thus, we treat them all together, using Fig. (1E) as our prototype. Note also that the argument does not depend on the order of the two arcs in series; e.g. it does not depend on which arc (4 or 6$)$ is connected to node D.

In this series connection, neither $\mathrm{C}_{4}$ nor $\mathrm{C}_{6}$ is equal to $\mathrm{C}_{\mathrm{CD}}$ Instead, $\mathrm{C}_{\mathrm{CD}}=\mathrm{C}_{4}+\mathrm{C}_{6}$. However the flow $\mathrm{U}_{\mathrm{CD}}=\mathrm{U}_{4}=$ $\mathrm{U}_{6}$.

If the conductivity functions of both arcs 4 and 6 are power law s, the series combination can be replaced by an equivalent single arc with conductivity function $\sigma_{\text {equivalent }}$, which is also power law $\mathrm{s}$,

$$
\sigma_{\text {equivalent }}=\sigma_{\text {equivalent }}(1)\left(\mathrm{C}_{\mathrm{CD}}\right)^{\mathrm{s}}
$$

where

$$
\left[\sigma_{\text {equivalent }}(1)\right]^{-1}=\left[\sigma_{4}(1)\right]^{-\mathrm{t}}+\left[\sigma_{6}(1)\right]^{-\mathrm{t}}
$$

For convenience we introduced $\mathrm{t}=(\mathrm{s}+1)^{-1}$

Thus, the effect of the two arcs in series between $\mathrm{C}$ and $\mathrm{D}$ is the same as a single arc between $\mathrm{C}$ and $\mathrm{D}$ with a conductivity function that has the same power law dependence on cost as before.

If either the original arc 4 or the added sixth arc (or both) has a different conductivity function (not simply a power s), then the series combination also does not have a simple power law s. This series connection produces the type of sixarc network which CK have shown can be arranged to have a BP Note, however, that it is equivalent to a five-arc $W B$ network in which one arc, arc 4, does not have the simple power-law s.

Because the CK theorem applies for any arbitrary arcs 4 and 6 (with the appropriate restrictions on power law dependence), in the final equivalent five arc network, arc 4 is also an arbitrary arc with the power law of the combination (power $\mathrm{s}$ if $\operatorname{arc} 4$ and $\operatorname{arc} 6$ each have power s, and not simply power $\mathrm{s}$ if either $\operatorname{arc} 4$, or arc 6 , or both are not simply power s).

\section{CONCLUSION: BRAESS PARADOX WITH ONLY FIVE ARCS}

We have now examined all nine possible ways to add a sixth arc to a five-arc $W B$ network, and have shown that for each way, if one can arrange six arcs to get a $\mathrm{BP}$, one can just as well arrange five arcs to get a BP; at least one of the five arcs must not have the same power law s as the others.

Since CK have shown that one such arc out of six is sufficient to be able to arrange for a $\mathrm{BP}$, the only conclusion is that one out of five is also sufficient, and (by CK theorems $11 \& 12$ ) that these five must be arranged in a $W B$ connection (Fig. 1A).

Thus, we have refined the CK theorem, which showed that six arcs (at least one of which does not have the same power law conductivity) with an embedded Wheatstone Bridge are sufficient to produce a Braess Paradox.

\section{Refined CK Theorem}

Five arcs (at least one of which does not have the same power law conductivity) arranged in a Wheatstone Bridge, are Necessary and Sufficient to produce a Braess Paradox in a two terminal network (i.e. a network with one origin and one destination).

\section{ACKNOWLEDGEMENTS}

We wish to thank Bruce Calvert and Grant Keady for several very useful discussions about the Braess Paradox and for their help in understanding the proofs of their theorems. We thank Robin Lindsey for many general interesting discussions, many helpful suggestions, and for introducing the unpublished work of Igal Milchtaich. We thank Donald McKenzie for helpful discussions about the Calvert and Keady theorems. We thank Gordon Davies and Kings College London for their hospitality when most of this work was done.

The research received some support from Gilora Assoc.

$$
\begin{aligned}
& \text { ABBREVIATIONS } \\
& \begin{array}{ll}
\mathrm{BP}= & \text { Braess Paradox } \\
\mathrm{C} & =\text { Link/arc Cost } \\
\mathrm{CK}= & \text { Calvert and Keady [2] } \\
\mathrm{GC}= & \text { Generalized Cost (see caption for Table } 1 \text { and see } \\
& \text { Dial [5] } \\
\mathrm{t} & =(\mathrm{s}+1)^{-1} \\
\mathrm{U} & =\text { Flow } \\
W B= & \text { Wheatstone Bridge } \\
\mathrm{V} & =\text { Number of pipes } \\
\sigma \quad= & \text { Conductivity function }
\end{array}
\end{aligned}
$$

\section{REFERENCES}

[1] D. Braess, "Ueber ein paradoxen der verkehrsplannung", Unternehmensforschung, vol. 12, pp. 258-268, 1968.

[2] B. Calvert and G. Keady, "Braess's paradox and power-law nonlinearities in networks", J. Austral. Math. Soc. Ser., vol. B 35, pp. 122, 1993.

[3] C.M. Penchina, "Power-Law congestion costs: minimal revenue pricing and the braess paradox", Open Transport. J., vol 2, pp. 4752, 2008.

[4] C.M. Penchina and L.J. Penchina, "The Braess paradox in mechanical, traffic, and other networks", Am. J. Phys., vol. 71(5), pp. 479-482, May 2003.

[5] R. Dial, "Minimal-revenue congestions pricing part I: A fast algorithm for the single-origin case", Transport. Res., vol. B33, pp. 189-202, 1999. 
[6] I. Milchtaich, "Network topology and the efficiency of equilibrium", Games Econ. Behav. vol. 57, pp. 321-346, 2006.

[7] C.M. Penchina, "Braess paradox: maximal penalty in a minimal critical network", Transport. Res., vol. A31(5), pp. 379-388, 1997.
[8] M. Frank, "The Braess paradox", Math. Program., vol. 20, pp. 283-302, 1981.

Received: March 31, 2008

Revised: April 28, 2008

Accepted: November 8, 2008

(c) Claude M. Penchina; Licensee Bentham Open

This is an open access article licensed under the terms of the Creative Commons Attribution Non-Commercial License (http://creativecommons.org/licenses/by$\mathrm{nc} / 3.0 /$ ) which permits unrestricted, non-commercial use, distribution and reproduction in any medium, provided the work is properly cited. 